\title{
Study of Baicalin toward COVID-19 Treatment: In silico Target Analysis and in vitro Inhibitory Effects on SARS-CoV-2 Proteases
}

\author{
Chingju Lin ${ }^{a}$ Fuu-Jen Tsaib,c, d Yuan-Man Hsu ${ }^{\mathrm{e}}$ Tsung-Jung Hof,g, h

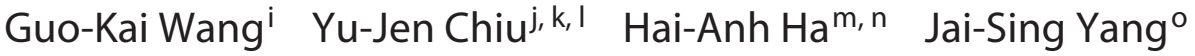

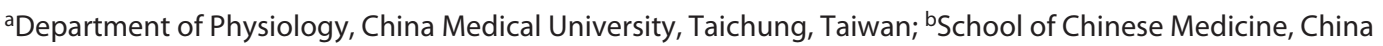
Medical University, Taichung, Taiwan; 'Department of Medical Research, Human Genetics Center, China Medical University Hospital 404, Taichung, Taiwan; 'Department of Medical Genetics, China Medical University Hospital, Taichung, Taiwan; 'Department of Biological Science and Technology, China Medical University, Taichung, Taiwan; fDepartment of Chinese Medicine, Hualien Tzu Chi Hospital, Buddhist Tzu Chi Medical Foundation, Hualien, Taiwan; IIntegration Center of Traditional Chinese and Modern Medicine, Hualien Tzu Chi Hospital, Buddhist Tzu Chi

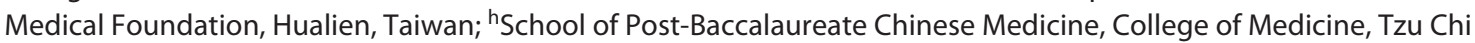
University, Buddhist Tzu Chi Medical Foundation, Hualien, Taiwan; 'School of Pharmacy, Anhui University of Chinese Medicine, Anhui Province Key Laboratory of Research \& Development of Chinese Medicine, Hefei, China; 'Division of Reconstructive and Plastic Surgery, Department of Surgery, Taipei Veterans General Hospital, Taipei, Taiwan; kDepartment of Surgery, School of Medicine, National Yang Ming University, Taipei, Taiwan; Institute of Clinical Medicine, National Yang-Ming University, Taipei, Taiwan; ${ }^{\mathrm{m} S}$ School of Pharmacy, China Medical University, Taichung, Taiwan; ${ }^{n}$ Faculty of Pharmacy, Duy Tan University, Da Nang, Vietnam; ${ }^{\circ}$ Department of Medical Research, China Medical University Hospital, China Medical University, Taichung, Taiwan
\end{abstract}

\section{Keywords}

Baicalin · Coronavirus disease-19 - SARS-CoV-2 .

3-Chymotrypsin-like cysteine protease · Papain-like protease

\begin{abstract}
Negative impacts of COVID-19 on human health and economic and social activities urge scientists to develop effective treatments. Baicalin is a natural flavonoid, extracted from a traditional medicinal plant, previously reported with anti-inflammatory activity. In this study, we used pharmacophore fitting and molecular docking to screen and determine docking patterns and the binding affinity of baicalin on 3 major targets of SARS-CoV-2 (3-chymotrypsin-like cysteine
\end{abstract}

karger@karger.com www.karger.com/bmh

Karger $\stackrel{\text { ' }}{5}$

GOPEN ACCESS
(C) 2021 The Author(s)

Published by S. Karger AG, Basel

This is an Open Access article licensed under the Creative Commons Attribution-NonCommercial-4.0 International License (CC BY-NC) (http://www.karger.com/Services/OpenAccessLicense), applicable to the online version of the article only. Usage and distribution for commercial purposes requires written permission. protease [3CLpro], papain-like protease [PLpro], and RNAdependent RNA polymerase). The obtained data revealed that baicalin has high pharmacophore fitting on $3 \mathrm{CL}$ pro and predicted good binding affinity on PLpro. Moreover, using the enzymatic assay, we examined the inhibitory effect of baicalin in vitro on the screened enzymes. Baicalin also exhibits inhibitory effect on these proteases in vitro. Additionally, we performed pharmacophore-based screening of baicalin on human targets and conducted pathway analysis to explore the potential cytoprotective effects of baicalin in the host cell that may be beneficial for COVID-19 treatment. The result suggested that baicalin has multiple targets in human cell that may induce multiple pharmacological effects. The result of pathway analysis implied that these targets may be associated with baicalin-induced bioactivities that are in-
Correspondence to:

Ha-Anh Ha, hahaianh@dtu.edu.vn

Jai-Sing Yang, jaisingyang@ gmail.com 
volved with signals of pro-inflammation factors, such as cytokine and chemokine. Taken together with supportive data from the literature, the bioactivities of bailalin may be beneficial for COVID-19 treatment by reducing cytokine-induced acute inflammation. In conclusion, baicalin is potentially a good candidate for developing new therapeutic to treat $\mathrm{CO}$ VID-19.

(c) 2021 The Author(s)

Published by S. Karger AG, Basel

\section{Introduction}

COVID-19 has been identified as a pandemic since March 2020; since then, billions of people around the world have suffered negative impacts of COVID-19 on human health as well as socioeconomic activities $[1,2]$. In response to the pandemic, preventive measures and clinical care are the main front-line battles for reducing morbidity and mortality. Recent vaccination roll-out in a number of countries is the most efficient measure for COVID-19 prevention. However, concerns are still rising about the emergence of new virus variants, uneven supply of vaccines, and little improvement in treatment discovery $[3,4]$. Since COVID-19 is a new disease, more research is urgently needed to find an effective treatment to cope with this public health crisis.

SARS-CoV-2, a single-strand RNA coronavirus is the pathogen of COVID-19 [5]. Based on the accumulated studies of pathogenesis of coronaviruses family, several molecular targets were selected for discovering therapeutics against SARS-CoV-2, namely, 3-chymotrypsin-like cysteine protease (3CLpro), papain-like protease (PLpro), and RNA-dependent RNA polymerase (RDRP) [6-8]. As these enzymatic proteins are crucial for virus replication, inhibiting these targets may reduce virus load, thereby inducing an antiviral effect [9]. In addition, the discovery of drugs intended to treat severe symptoms of COVID-19 is of interest to laboratories [6]. The progression of COVID-19 is associated with acute inflammation and immune response that may cause hyper-inflammatory syndrome, referred as the "cytokine storm" (CS) [10]. The molecular mechanism behind a CS is known as the excessive releasing of pro-inflammatory molecules, such as IL1, IL-6, and TNF- $\alpha$ [11]. Therefore, the CS can be dismissed by inhibiting the cytokine amplification or blocking cytokine-induced signaling cascade inside the host cell [12].

Baicalin is a bioactive natural compound, isolated from the medicinal plant Scutellaria baicalensis (SB), a commonly used plant in traditional Chinese medicine
[13]. Extract of SB has been reported with anti-inflammation $[14,15]$, antioxidant $[13,16]$, or antiviral $[17,18]$ activities that possibly conferred by its major chemical compositions, such as baicalin and baicalein. Recently, SB and several herbal formulas comprising SB were suggested as potential therapeutics to treat COVID-19 with supportive data provided from in silico approaches or system pharmacological studies [7, 19-22]. Baicalin is known to be responsible for the major biological effects of SB and exhibits anti-inflammatory and antiviral effects with low cytotoxicity [23-25]. Baicalin is the main composition of Flavocoxid, an approved medical food, which is classified as generally recognized as safe by regulatory requirement. A recent repaper reported that, Flavocoxid has low risk and fewer side effects than nonsteroidal antiinflammatory drugs [26]. Recent reviews mentioned baicalin as a potential compound to develop as COVID-19 therapeutic [21, 27, 28]. However, in order to get closer to the drug development, more evidence and insight is needed about its possible effects on the molecular targets toward COVID-19 treatment.

In this study, we computationally screened and determined potential binding patterns of baicalin on 3 major targets of SARS-CoV-2 (3CLpro, PLpro, and RDRP) and examined the inhibitory effect of baicalin in vitro on the screened enzymes. Furthermore, we performed pharmacophore-based screening of baicalin on human targets and conducted pathway analysis to explore the potential cytoprotective effects of baicalin in the host cell that may also be beneficial for COVID-19 treatment.

\section{Material and Methods}

Structure Preparation for in silico studies

The structures of 3CLpro (code: 6W63), PLpro (code: 6WX4), and RDRP (code: 7BV2) of SARS-CoV-2 were obtained from the Protein Data Bank (rcsb.org) [29]. The protein preparation steps, including fixing the common structure problems and applying protonation status at $\mathrm{pH} 7.4$ were processed before docking using integrated tools of BIOVIA, Dassault Systèmes, Discovery Studio 2020, San Diego, CA, USA: Dassault Systèmes, 2020. The chemical features of baicalin was sketched using BIOVIA Draw, followed by the preparation for generating the appropriate protonated isomers and tautomers at $\mathrm{pH} 7.4$ as described previously [28].

\section{In silico Target Screening of Baicalin for SARS-CoV-2 by}

Pharmacophore Models

Pharmacophore models for molecule interactions were created according to the complexities of the prepared proteins and their in situ ligands as previously described [28]. Each model was also confirmed with the known inhibitors [29-31]. The sensitivity of the pharmacophore model for 3CLpro was 0.73913 (true-positive/to- 
tal active: 17/23), while the sensitivities of the pharmacophore model for PLpro and RDRP were 0.5 (true-positive/total active: $1 / 2$ ), and 0.75 (true-positive/total active: $3 / 4$ ), respectively. Pharmacophore mapping was conducted using the provided tool of DS 2020 system on 3CLpro, PLpro, and RDRP. The pharmocophore fit value of each ligand in the target was analyzed from the previous pharmacophore mapping result, using the intergrated "Ligand Profiler" function of the software system.

Molecular Docking for Baicalin on 3CLpro, PLpro, and RDRP

The baicalin-binding sites on 3Clpro, PLpro, and RDRP of SARS-CoV-2 were identified according to the native co-crystal ligand of the download PDB model. The docking optimization module, CDOCKER protocol embedded in DS 2020 system, was used for the docking process as previously described [28]. To check the accuracy of the docking algorithm, the native inhibitory ligand was used for re-docking and calculating root mean square deviation of the top docking poses to the co-crystalized ligand. The result is presented in online supplementary Table 1 (for all online suppl. material, see www.karger.com/doi/10.1159/000519564).

The 3-dimensional (3D) structure of baicalin was downloaded from the PubChem database. After energy minimization, baicalin was docked into 3CLpro, Plpro, or RDRP. The docking result of each target was ranked by the "CDOCKER energy" value to identify the most potential binding pose (lowest binding energy). Docking results were then scored by LigScorel using the intergrated tool "Score Ligand Poses" to obtain the final docking score value. The binding pattern and ligand-receptor interactions were analyzed visually in $3 \mathrm{D}$ view and drawn into 2 -dimensional diagram as the final result.

\section{Enzymatic Activity and Inhibition Assays}

The enzyme activity assays was performed as described previously [32]. Briefly, the protease activity of 3CLpro of SARS-CoV-2 virus was measured by a continuous kinetic assay, with the substrate MCA-AVLQSGFR-Lys (Dnp)-Lys-NH2 (GL Biochem, Shanghai), using wavelengths of $320 \mathrm{~nm}$ and $405 \mathrm{~nm}$ for excitation and emission, respectively. Similarly, the protease activity of PLpro of SARS-CoV-2 virus was also measured with the substrate Ubiquitin-AMC.

\section{Pathway Analysis}

KEGG pathway analysis (accessible at www.kegg.jp) was used in this study, following the previous guidelines [33]. From the pharmacophore fitting result, with possible targets of baicalin in human cell, and the associated KEGG-IDs were collected and submitted into the KEGG Mapper Web page as a single gene list. The mapping process was performed through the Web interface. The default target pathway database was applied to perform the analysis. Based on the integrated algorithm of KEGG, a list of relevant pathways was then generated by the KEGG system. Results of pathways were annotated with KEGG Orthology identifiers as set by default and shown in the pathway tab by the Web-based system for an on-site view and download for further analysis.

\section{Statistical Analysis}

For each in vitro study, 3 independent experiments were conducted. Statistical analysis was performed using 1-way ANOVA followed by Dunnett's post hoc test. ${ }^{* * *} p<0.001$ versus untreated control.

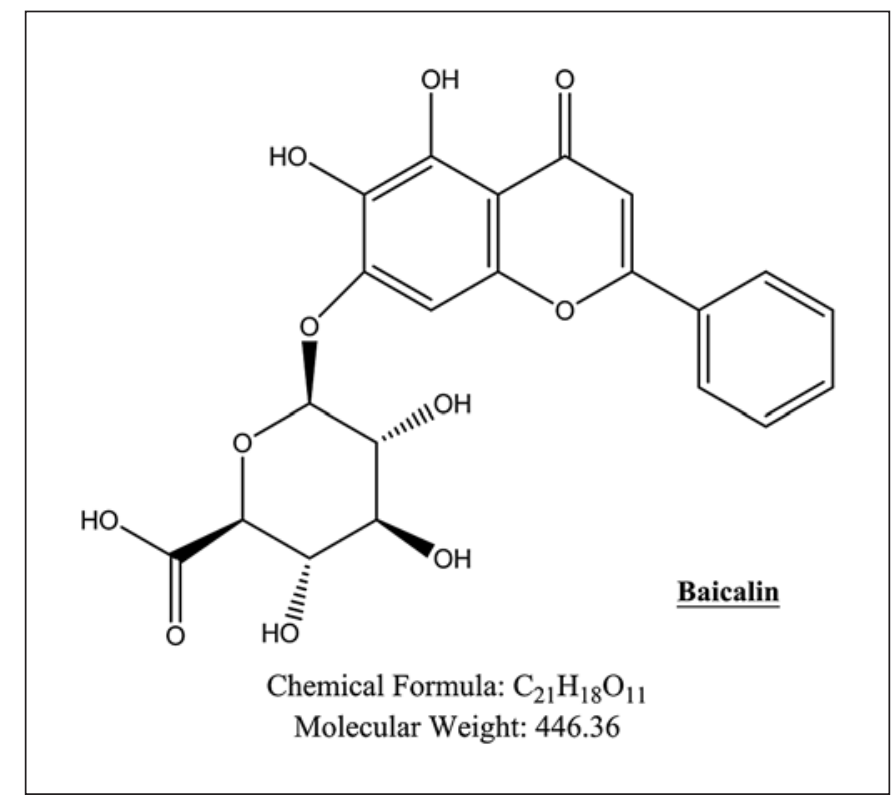

Fig. 1. Chemical structure of baicalin.

\section{Results}

\section{In silico Target Analysis of Baicalin with 3CLpro, PLpro, and RDRP}

The flavonoids class has been regarded as a promising therapeutic strategy against SARS-CoV-2 infection due to its wide spectrum of biological properties, including antioxidant, anti-inflammatory, and antiviral activities. We examined whether baicalin (molecular formula: $\mathrm{C}_{21} \mathrm{H}_{18} \mathrm{O}_{11}$, Fig. 1), one of the major flavonoids in the traditional Chinese medicinal herb "Huang qin" also exerts an antiviral activity against SARS-CoV-2 infection. The flow diagram illustrating the research design is shown in Figure 2. After screening 3 possible targets of baicalin for SARS-CoV-2, the in silico assay was performed to determine the binding affinity between baicalin and its potential targets, followed by in vitro protease activity assays. We used pharmacophore models in PharmaDB to determine binding affinities of baicalin plus 3 other positive-control antiviral drugs (remdesivir and lopinavir and ritonavir) to 3CLpro, PLpro, and RDRP of SARS-CoV-2. Among antiviral drugs, remdesivir has been shown to inhibit RDRP, while lopinavir and ritonavir suppress the main protease protein 3CLpro polyprotein of SARS-CoV-2 $[9,34]$. The in silico assay results in Table 1 suggested a higher pharmacophore fit value of baicalin (fitting value: 0.726 ) on 3CLpro than that of lopinavir (0.608) and ritonavir (0.654). However, the docking score result showed a lower score for baicalin (LigScore: 4.47) 


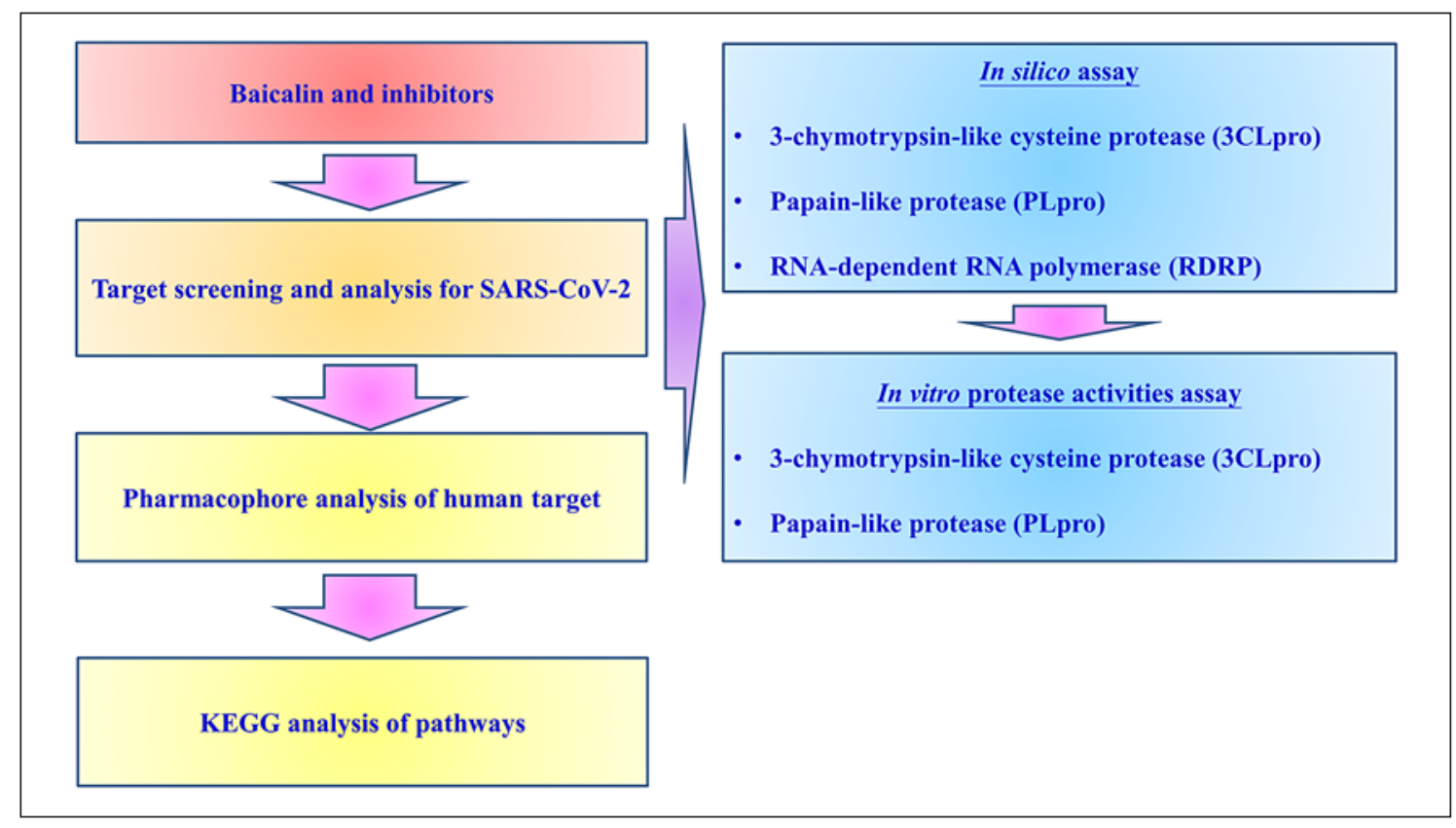

Fig. 2. Experimental design of the current study to assess the effect of baicalin against a SARS-CoV-2 infection in silico study. Details are discussed in the text. RDRP, RNA-dependent RNA polymerase; PLpro, papain-like protease; 3CLpro, 3-chymotrypsin-like cysteine protease.

Table 1. The pharmacophore fitting and LigScore results of baicalin and antiviral agents on 3CLpro, PLpro, and RDRP of SARS-CoV-2

\begin{tabular}{|c|c|c|c|c|c|c|}
\hline \multirow[t]{2}{*}{ Compound name } & \multicolumn{2}{|l|}{ 3CLpro } & \multicolumn{2}{|l|}{ PLpro } & \multicolumn{2}{|l|}{ RDRP } \\
\hline & fitting value & LigScore & fitting value & LigScore & $\begin{array}{l}\text { fitting } \\
\text { value }\end{array}$ & LigScore \\
\hline Remdesivir & - & - & - & - & 0.730 & 6.62 \\
\hline Lopinavir & 0.608 & 6.59 & - & - & - & - \\
\hline Ritonavir & 0.654 & 5.98 & - & - & - & - \\
\hline Baicalin & 0.726 & 4.47 & 0.573 & 5.64 & 0.453 & 6.49 \\
\hline
\end{tabular}

3CLpro, 3-chymotrypsin-like cysteine protease; PLpro, papain-like protease; RDRP, RNA-dependent RNA polymerase.

than that of lopinavir (6.59) and ritonavir (5.98). On the PLpro target, baicalin demonstrated more promising data with a pharmacophore fitting value (0.573) greater than 0.5 and a LigScore value (5.64) $>5$. In addition, the in silico screening result on RDRP of baicalin showed both pharmacophore fitting (0.453) and LigScore (6.49) lower than those of remdesivir (0.730 and 6.62, respectively).

Binding patterns of baicalin on the selected targets were then analyzed from the obtained molecular docking result. $3 \mathrm{D}$ and 2-dimensional ligand interaction diagram results on 3CLpro (Fig. 3), PLpro (Fig. 4), and RDRP (Fig. 5) were shown for a more in-depth understanding of the intermo- lecular interactions between baicalin and 3CLpro, PLpro, and RDRP of SARS-CoV-2. According to bond interaction statistics (Table 2), baicalin formed multiple bond interaction with residues HIS41, CYS44, LEU141, ASN142, GLY143, CYS145, HIS163, GLU166, and MET165 of 3CLpro, and the interactions included 11 hydrogen bonds and 4 hydrophobic interactions. In addition, baicalin formed multiple bond interactions with residues CYS111, LEU162, GLY163, TYR264, and GLY271 of PLpro, with 9 hydrogen bonds and 1 hydrophobic interaction. For RDRP, baicalin also formed bond interactions with residues LYS545, Arg555, ASN691, and ASP760 of RDRP, and it consisted of 


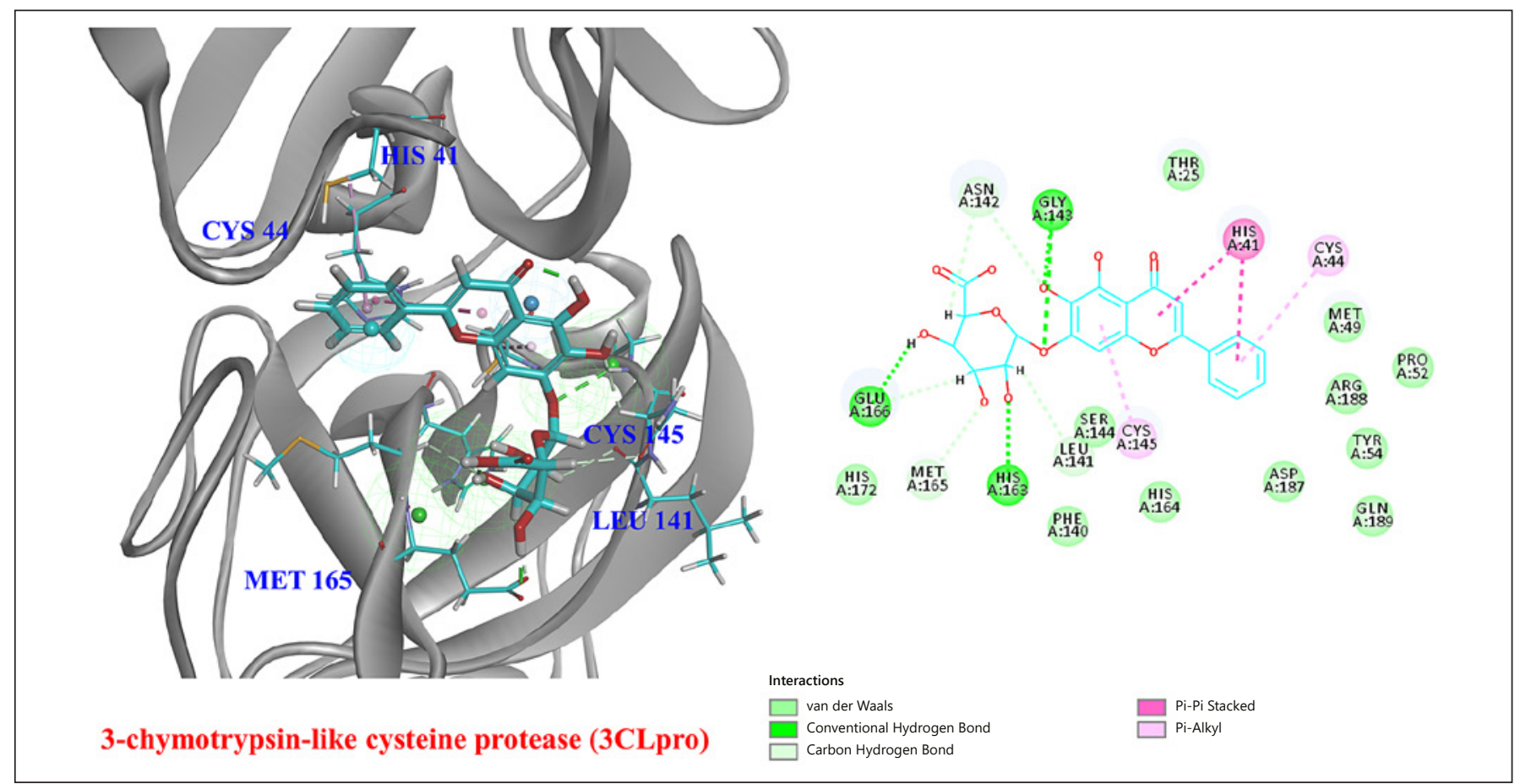

Fig. 3. Molecular docking model of baicalin binding to 3CLpro. The left panel shows the 3D ligand interaction model of selective binding sites between baicalin and 3CLpro using Discovery Studio 2021. The right panel shows the $2 \mathrm{D}$ ligand interaction diagram be-

11 hydrogen bonds and 2 hydrophobic interactions. In comparison among these docking patterns, the intermolecular interactions of ritonavir, lopinavir, or baicalin with 3CLpro showed that all 3 compounds shared the same binding pocket, and the baicalin-3CLpro interaction consists of more residue involvements than the others (Fig. 3, 6). In addition, among the 3 drug targets, baicalin showed the highest fitting value but the lowest docking score to 3CLpro, followed by PLpro and RDRP (Table 1).

\section{Inhibition of 3CLpro and PLpro Protease Activities by Baicalin}

Since baicalin exhibited high binding affinity to 3CLpro and PLpro, we next determined whether baicalin could inhibit the protease activities of 3CLpro and PLpro. The in vitro protease activity assay demonstrated that the activity of 3CLpro was effectively attenuated by baicalin at 50,100 , and $200 \mu \mathrm{M}$ in a concentration-dependent manner (Fig. 7). In addition, baicalin also exerted significant inhibition on PLpro activity at 12.5, 25, 50, 100, and $200 \mu \mathrm{M}$ in a dose-dependent manner (Fig. 8). Our data indicated that the activity of PLpro protease was more sensitive to baicalin treatment than that of 3 CLpro. The $\mathrm{IC}_{50}$ values of tween baicalin and 3CLpro. Different bond interaction forces are illustrated with different colors as indicated in the figure. 3D, 3-dimensional; 2D, 2-dimensional; 3CLpro, 3-chymotrypsin-like cysteine protease. baicalin and positive controls (GC376 and HY-17542) on protease activities are presented in Table 3.

\section{KEGG Pathway Analysis Result}

The KEGG pathway analysis result showed the possible effects of baicalin on certain targets in the view of relevant cellular signaling pathway. In the "Coronavirus disease - COVID-19" pathway (shown in Fig. 9), the targets detected by the KEGG mapper tool were mitogen-activated protein kinase (MAPK), spleen-associated tyrosine kinase (SYK), and phosphatidylinositol-3-kinase (PI3K), involved in the IgG-induced signal cascade. Besides, interleukin-1 receptor-associated kinase is also found as possible target that involved in the toll-like receptor $2 / 4$ signaling pathway. The MAPK pathway is also analyzed in more detail (shown in Fig. 10), which showed the involvements of interleukin-1 receptor-associated kinase $1 / 4$ and the phosphorylation cascade of p38 and MAPK, following the signal transduction after IL-1 bound to IL1R. In the "chemokine signaling pathway" (shown in Fig. 11), targets detected by the KEGG mapper tool were PI3K and GSK3 (glycogen synthase kinase 3) which involved both cytokine production and cell survival. 

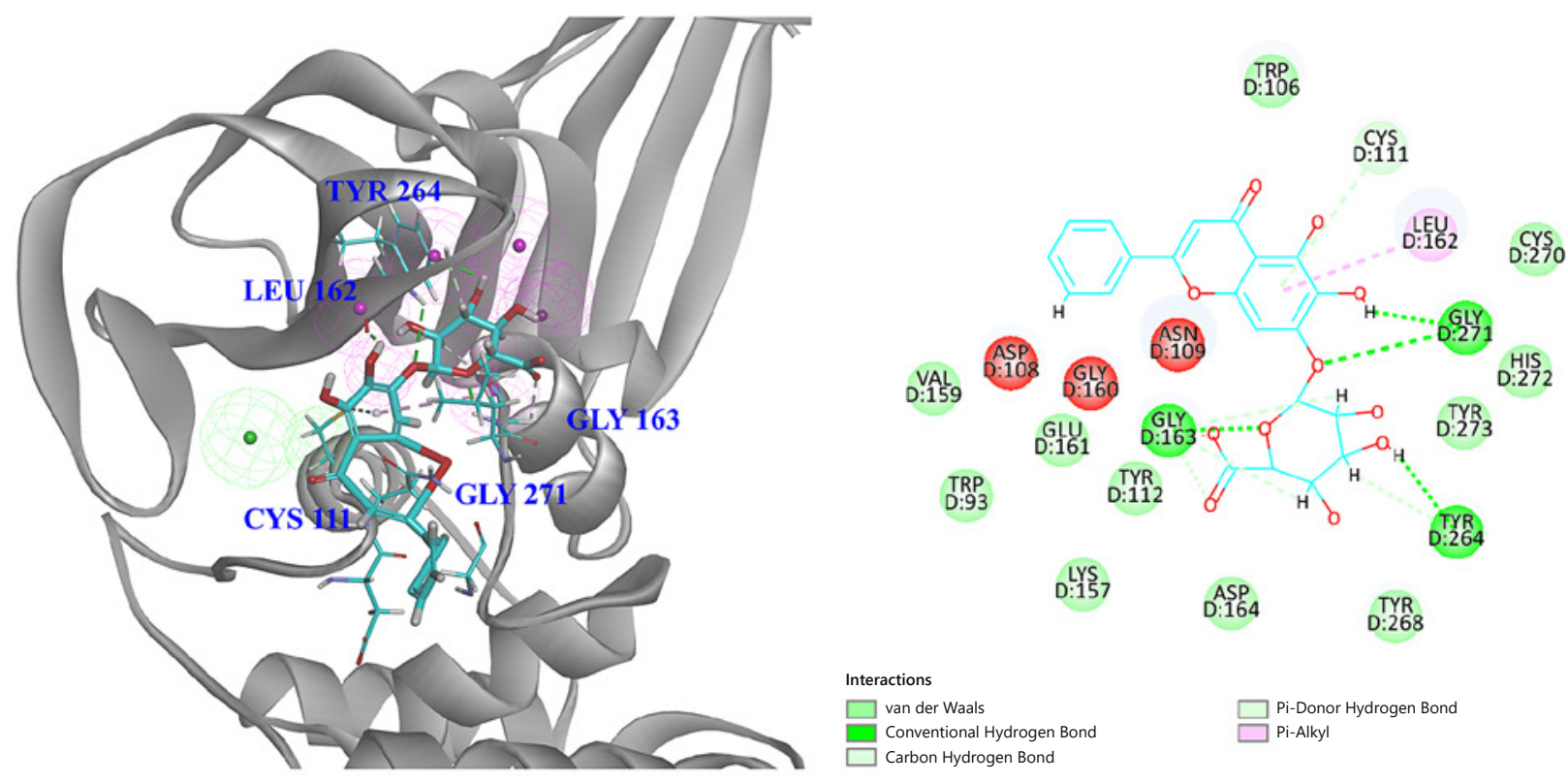

Papain-like protease (PLpro)

Fig. 4. Molecular docking model of baicalin binding to PLpro. The left panel shows the 3D ligand interaction model of selective binding sites between baicalin and PLpro using Discovery Studio 2021. The right panel shows the $2 \mathrm{D}$ ligand interaction diagram between baicalin and PLpro. Different bond interaction forces are illustrated with different colors as indicated in the figure. 3D, 3-dimensional; 2D, 2-dimensional; PLpro, papain-like protease.

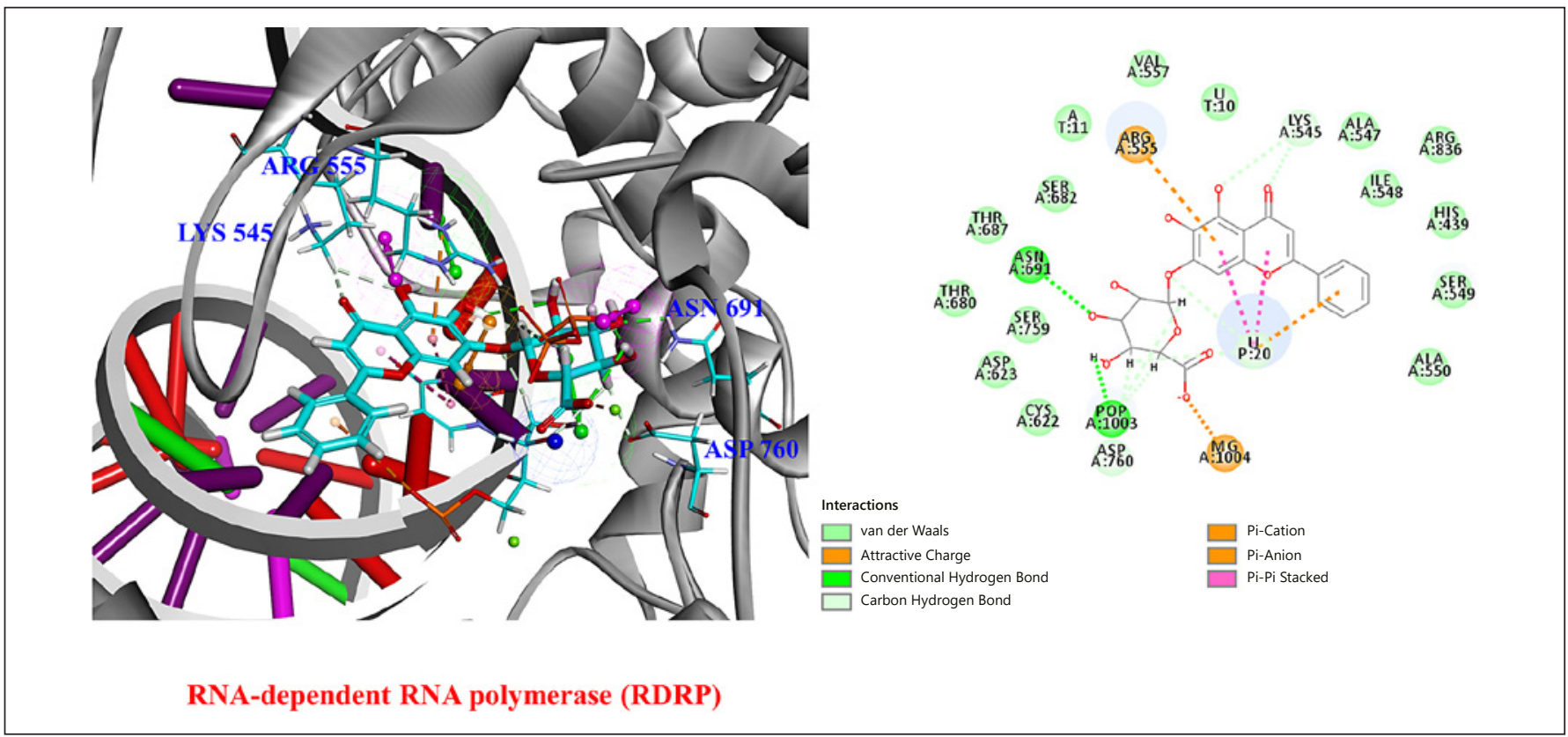

Fig. 5. Molecular docking model of baicalin binding to RDRP. The left panel shows the 3D ligand interaction model of selective binding sites between baicalin and RDRP using Discovery Studio 2021. The right panel shows the $2 \mathrm{D}$ ligand interaction diagram between baicalin and RDRP. Different bond interaction forces are illustrated with different colors as indicated in the figure. 3D, 3-dimensional; 2D, 2-dimensional; RDRP, RNA-dependent RNA polymerase. 


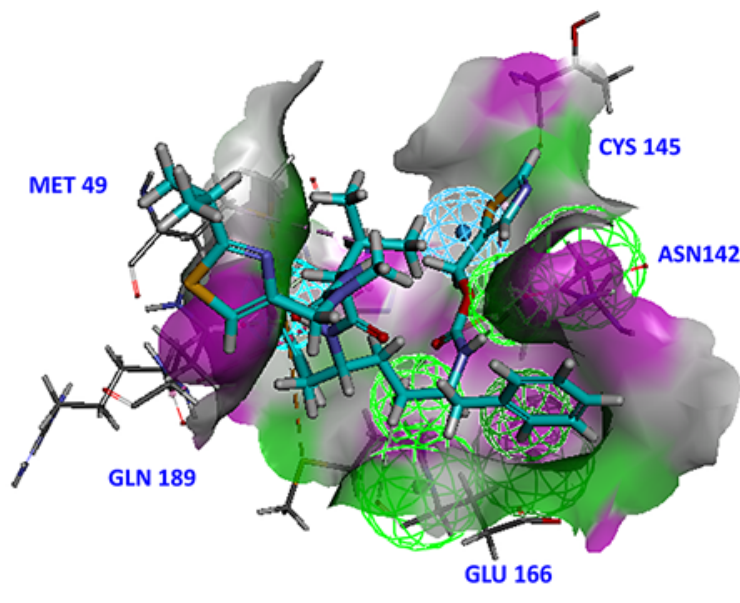

Ritonavir/3CLpro

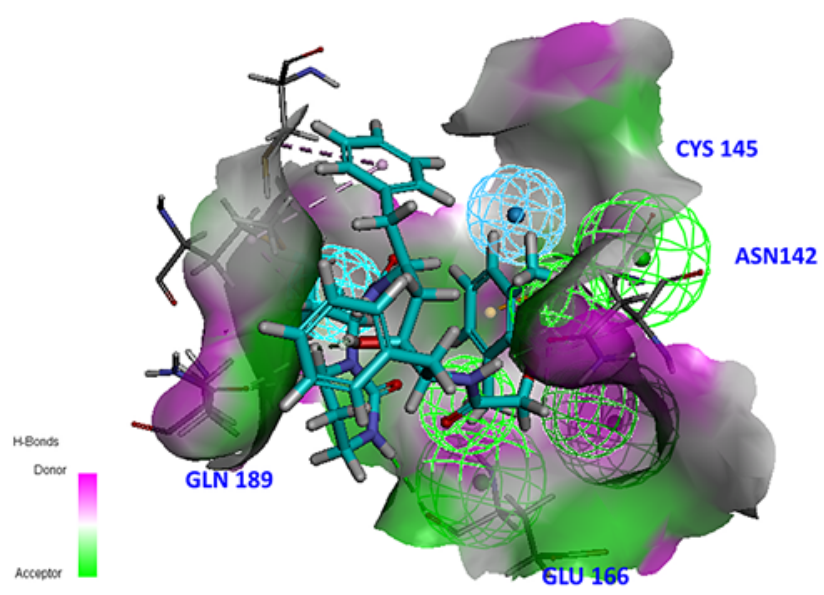

Lopinavir/3CLpro

\begin{tabular}{|c|c|}
\hline $\begin{array}{l}\text { Target of 3-chymotrypsin-like cysteine protease } \\
\text { (3CLpro) }\end{array}$ & Interaction Residues \\
\hline Baicalin & HIS41 、CYS44、LEU141 、ASN142、GLY143、CYS145、HIS163、GLU166、MET165 \\
\hline Ritonavir & MET49, ASN142, CYS145, GLU166, PRO168, GLN189, THR190 \\
\hline Lopinavir & THR26、HIS41 、 MET49、ASN142、CYS145、GLU166 、 GLN189 \\
\hline
\end{tabular}

Fig. 6. Molecular docking model of ritonavir and lopinavir binding to 3CLpro. The 3D ligand interaction models at the binding site, between ritonavir (upper left) and lopinavir (upper right) on 3CLpro using Discovery Studio 2021. Lower panel shows statistics of interaction residues between ligands (baicalin, ritonavir, and lopinavir) in the binding site of 3CLpro. 3D, 3-dimensional; 3CLpro, 3-chymotrypsin-like cysteine protease.

Table 2. The interaction statistics between the baicalin and with 3CLpro, PLpro, and RDRP of SARS-CoV-2

\begin{tabular}{llll}
\hline Target & \multicolumn{2}{l}{ H-bonds, } \\
& $n$ & $\begin{array}{l}\text { Hydro- } \\
\text { phobics, } n\end{array}$ & Interaction residues \\
\hline 3CLpro & 11 & 4 & HIS41, CYS44, LEU141, ASN142, GLY143, CYS145, HIS163, GLU166, and MET165 \\
PLpro & 9 & 1 & LEU162, GLY163, GLY271, TYR264, and CYS111 \\
RDRP & 11 & 2 & ASN691, LYS545, ASP760, and ARG555
\end{tabular}

3CLpro, 3-chymotrypsin-like cysteine protease; PLpro, papain-like protease; RDRP, RNA-dependent RNA polymerase.

\section{Discussion}

The 3 enzymes taken into this study, 3CLpro, PLpro, and RDRP, are characterized as highly conserved structures [35] as there is no report about the mutation in new emerging variants of SARS-CoV-2. Therefore, developing COVID-19 therapeutics targeting these enzymes is taking interest from laboratories. Approved antiviral drugs, such as lopinavir and ritonavir, showed consistency of in silico and in vitro data for the inhibitory effect on 3CLpro [36]. 
Fig. 7. Inhibitory effects of baicalin on the 3CLpro activity. The enzymatic and inhibition activity assay showed that baicalin inhibited 3CLpro activity at the concentrations of $12.5 \mu \mathrm{M}, 25 \mu \mathrm{M}, 50 \mu \mathrm{M}, 100 \mu \mathrm{M}$, and $200 \mu \mathrm{M}$ in a concentration-dependent manner. All data are shown as mean \pm SD from 3 independent experiments. ${ }^{* * *} p<$ 0.001 versus untreated control. 3CLpro, 3-chymotrypsin-like cysteine protease.
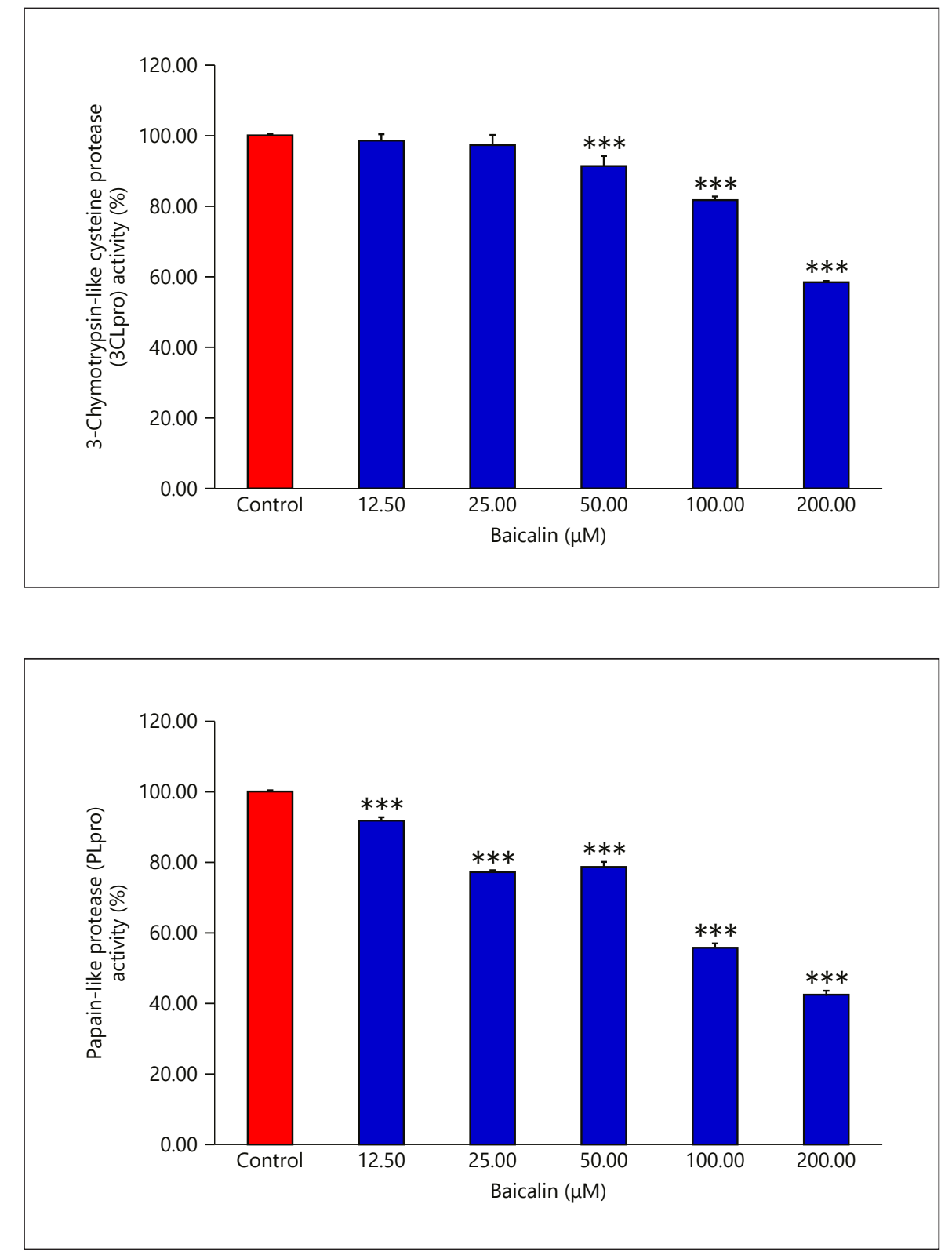

Fig. 8. Inhibitory effects of baicalin on PLpro activity. The enzymatic and inhibition activity assay showed that baicalin inhibited PLpro activity at the concentrations of $12.5 \mu \mathrm{M}, 25 \mu \mathrm{M}, 50 \mu \mathrm{M}, 100 \mu \mathrm{M}$, and $200 \mu \mathrm{M}$ in a concentration-dependent manner. All data are shown as mean \pm SD from 3 independent experiments. ${ }^{* * *} p<$ 0.001 versus untreated control. PLpro, papain-like protease.
Here, we examined the binding affinity of baicalin on the proteases (3CLpro and PLpro) and RDRP of SARS-CoV-2 by pharmacophore fitting and molecular docking. The pharmacophore mapping result showed that baicalin may be fit within the binding site of 3CLpro (Table 1). In addition, the molecular docking results are explicable with the formation of multiple bonds between ligand (baicalin) and the side chain residues at the binding cavity (Table 2). It also suggested that baicalin could interact with 3CLpro at the binding site to form a ligand-protein complex. The binding site of baicalin on the proteases (shown in Fig. 3-5) is similar to that of suggested inhibitors from previous
Table 3. The half maximal inhibitory concentration $\left(I C_{50}\right)$ of baicalin, GC376, and HY-17542 on protease activities

\begin{tabular}{lll}
\hline Compound & 3CLpro & PLpro \\
\hline Baicalin, $\mu \mathrm{M}$ & $>200$ & 177.6 \\
GC376 (positive control of 3CLpro), $\mu \mathrm{M}$ & 0.0087 & $\mathrm{ND}$ \\
HY-17542 (positive control of PLpro), $\mu \mathrm{M}$ & $\mathrm{ND}$ & 1.73 \\
\hline
\end{tabular}

Protease activities were monitored as a time-course measurement of the increase in fluorescence signal from fluorescently labeled peptide substrate, and the initial linear portion of slope (signal/min) was analyzed. ND indicates compound not tested against enzyme. 3CLpro, 3-chymotrypsin-like cysteine protease; PLpro, papain-like protease. 


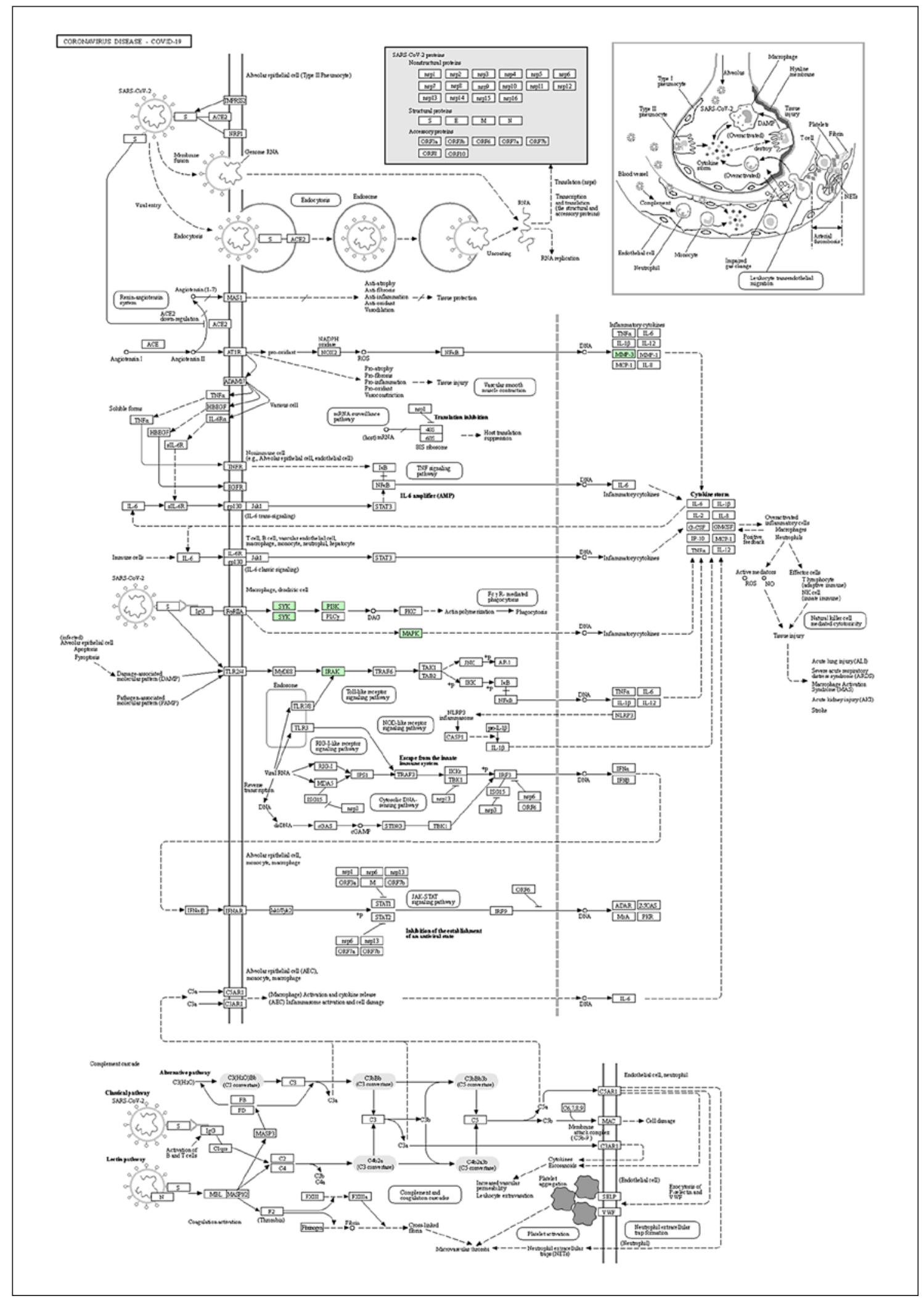

Fig. 9. Targets of baicalin (annotated with green color) in the pathway "Coronavirus disease - COVID-19 - Homo sapiens (human).” KEGG pathway ID: hsa05171. KEGG copyright permission ID: 210346. 


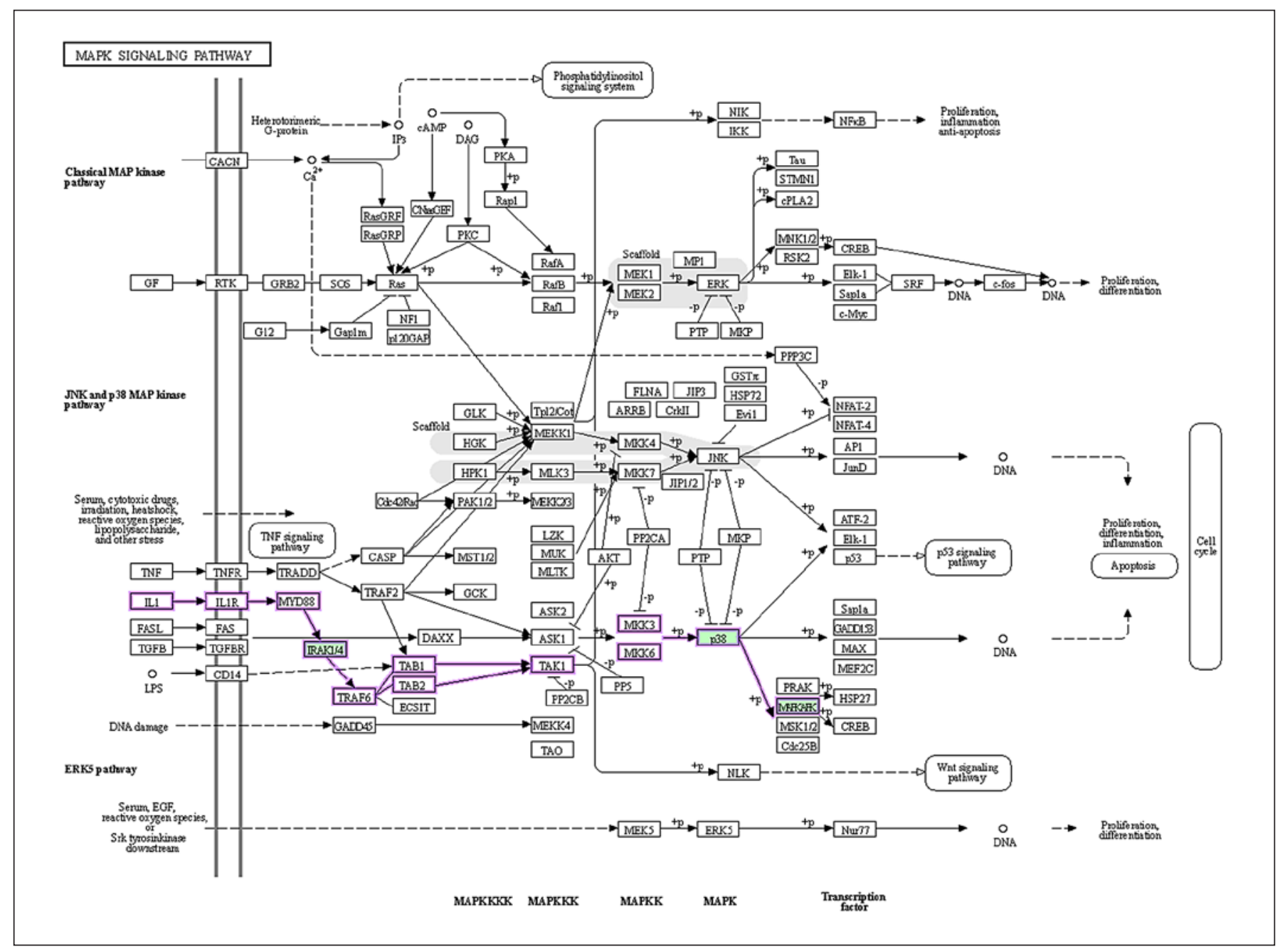

Fig. 10. Targets of baicalin (annotated with green color) in the pathway "MAPK signaling pathway - Homo sapiens (human).” KEGG pathway ID: hsa04010. KEGG copyright permission ID: 210346.

studies [7, 8, 37]. As reported by previous studies, these binding modes may confer an inhibitory effect by occupying catalytic cavity of enzymes [35]. However, with a low LigScore value, the intermolecular affinity of baicalin to 3CLpro was predicted lower than that of the clinical antiviral drugs ritonavir and lopinavir as previously defined for the algorithm of scoring function [38]. Consequently, the stability of the baicalin-3CLpro complex may not stable due to attributes of the van der Waals interaction, the polar attraction, and the desolvation penalty [38].

A study on baicalin and its aglycon, baicalein, showed that both compounds exert in vitro inhibitory effects on the RDRP of SARS-CoV-2; however, baicalin exhibited low potency than baicalein [39]. In this study, the pharmacophore fitting value of baicalin on RDRP received lower value than that of the other targets and below 0.5
(Table 1). Moreover, both the pharmacophore assay result and docking score of baicalin were found lower than those of remdesivir. Furthermore, the interaction statistics between baicalin and RDRP displayed only with 4 residues involved (Table 2), lower than that of 3CLpro and PLpro. These results suggested that the intermolecular interactions between baicalin and RDRP may not strong enough to establish stable binding and showed consistency with the previous study [39]. Therefore, we only selected 3CLpro and PLpro for in vitro experiments.

In vitro experiments were conducted for investigation of enzymatic inhibition effects on selected target proteins. We found that baicalin can inhibit the enzymatic activity of 3CLpro and PLpro (shown in Fig. 7, 8) as suggested by in silico data. However, the inhibitory effect of baicalin on 3CLpro $\left(\mathrm{IC}_{50}>200 \mu \mathrm{M}\right.$ ) is relative weaker than that on 


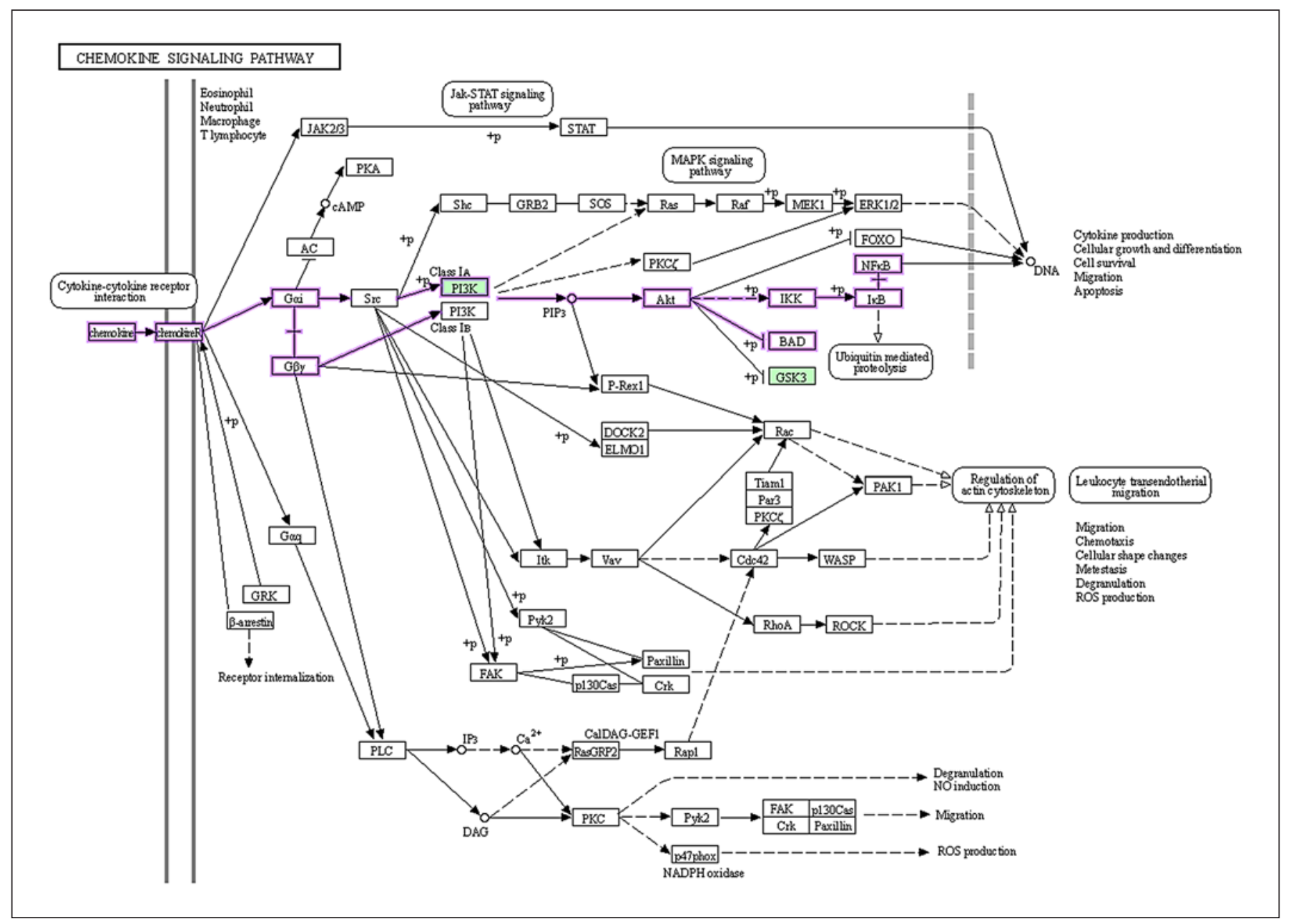

Fig. 11. Targets of baicalin (annotated with green color) in the pathway "Chemokine signaling pathway - Homo sapiens (human).” KEGG pathway ID: hsa04062. KEGG copyright permission ID: 210346.

PLpro $\left(\mathrm{IC}_{50}=178 \mu \mathrm{M}\right)$. Previous studies using different in vitro methods showed that baicalin has a good inhibitory effect on 3CLpro [7,20]. However, to the best of our knowledge, studies investigating the inhibitory effect of baicalin on PLpro have not been carried out. Therefore, our results may contribute as a reference when evaluating baicalin as an inhibitor of SARS-CoV-2 protease in future studies.

In addition to the investigation of the inhibitory effect on viral replication, we sought to uncover the possible effects of baicalin on the host cell. We investigated the possible targets of baicalin in human cells by a pharmacophore-based screening. Our results (shown in Table 4) revealed a set of target genes which were regulated following baicalin treatment. This result is consistent with previous reports that baicalin has a broad spectrum of biological effects [13]. Among those human targets, many of them are enzymes like oxidoreductases or transferases that partici- pate in cellular oxidative activities. The finding is in accordance with the previous reported anti-oxidative activities of baicalin [40]. A previous review also indicated that flavonoids including baicalin possibly inhibit SARS-CoV-2 due to their antioxidant and antiviral functions [21]. Furthermore, the literature indicated that lowering oxidative stress may reduce the risk of complications in COVID-19 that associated with the development of a CS [41].

A recent study on 5 flavones of SB using the structural biology approach, revealed dual function of baicalin, including inhibitory effect against enzymatic activity of SARS-CoV-2 3CLpro and protective effect against respiratory damage [42]. In this study, pathway analyses were carried out in order to construct a better insight of potential baicalin-induced effects on important targets that may protect the cell against cytokine-induced inflammation. "Coronavirus disease - COVID-19 pathway" and "MAPK 

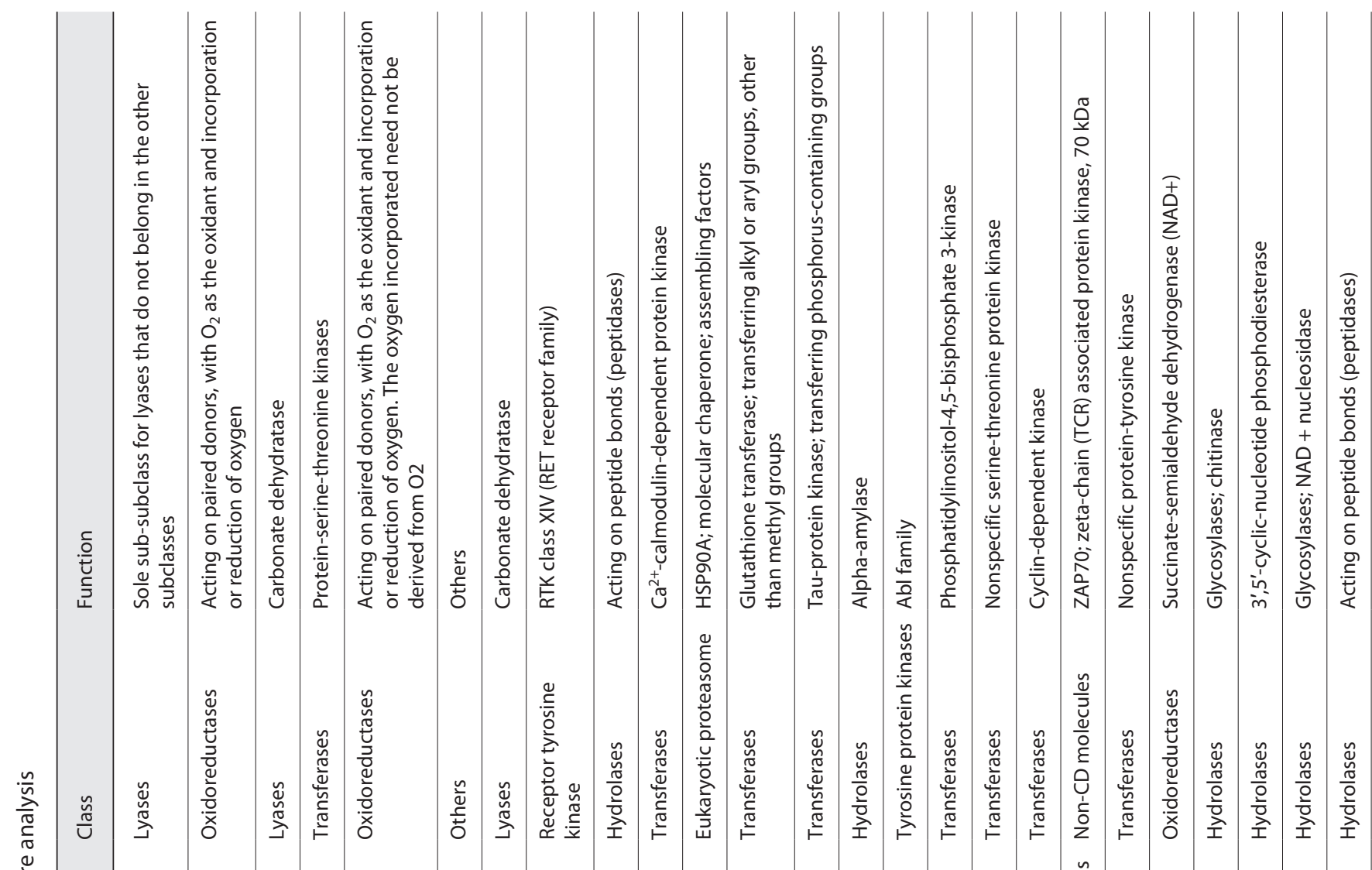

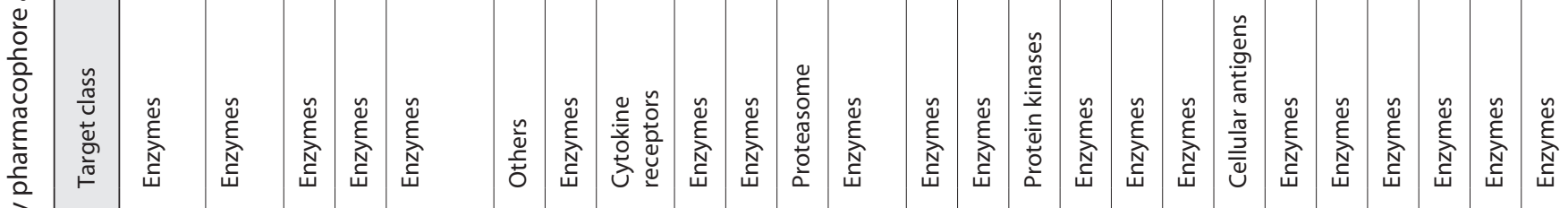

ลิ

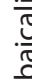

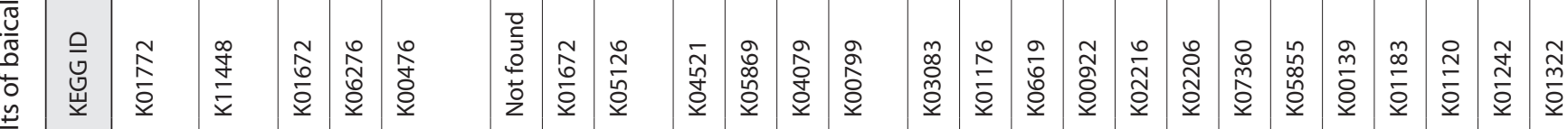

节

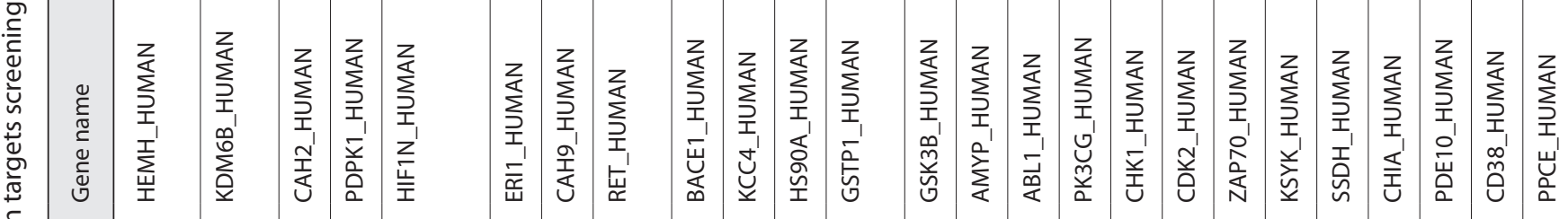

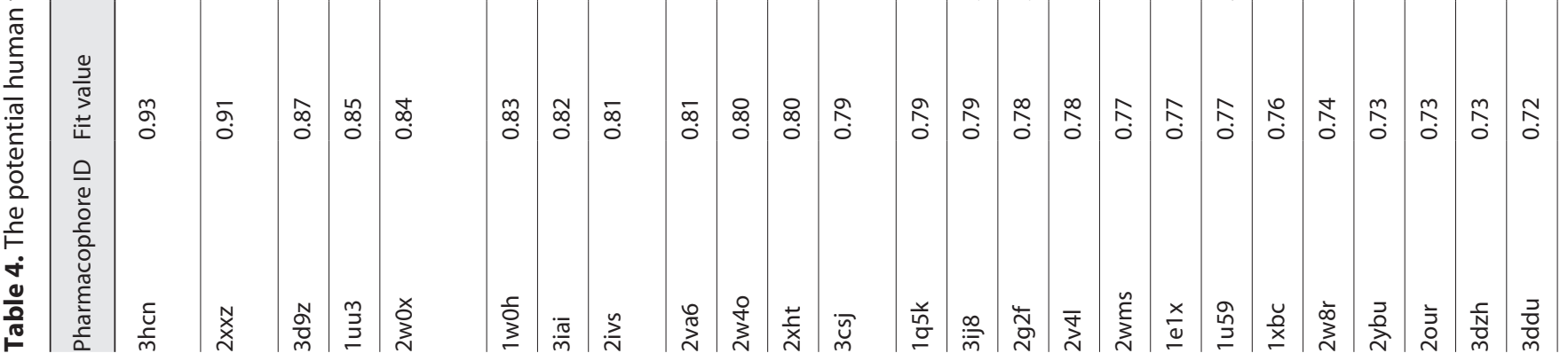




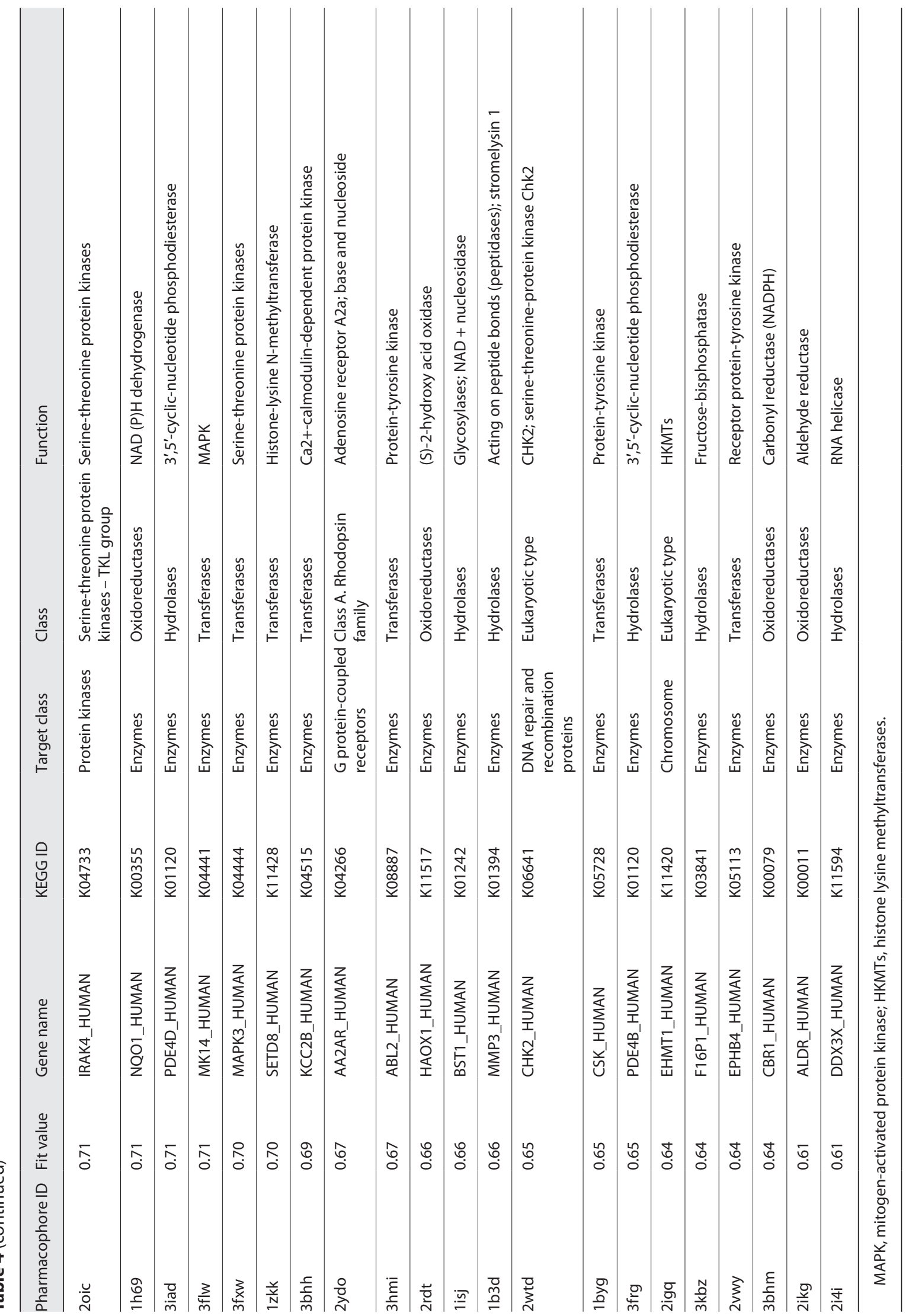


signaling pathways" (shown in Fig. 9, 10) indicated involvements of MAPK, SYK, and PI3K as targets of baicalin. This result is in accordance with previously reported studies that baicalin inhibits p38 MAPK, and this mechanism may be responsible for its anti-inflammation effect $[23,25$, $43,44]$. MAPK is activated under the stress of cytokine; therefore, inhibiting MAPK is a promising approach for hyper-inflammatory disease, such as COVID-19 [45]. Last, analysis on the pathway of "chemokine signaling" revealed involvements of PI3K and GSK3 as targets of baicalin. These 2 targets serve in the signal cascade, induced by chemokine after interacting directly with its specific receptor on the surface of the cell. The chemokine, as a pro-inflammatory factor, triggers signal to activate NF- $\mathrm{KB}$ and eventually promotes cytokine production. The produced cytokines can be released and cause further cycles of cytokine amplification that is harmful in acute inflammation condition, such as in COVID-19. According to previous reports, baicalin regulates the PI3K/AKT signal [24] and NF- $\mathrm{BB}$ $[23,43]$, and these activities may be responsible for baicalin-induced anti-inflammatory effect [16, 23, 43]. Our analysis and the supportive data from the literature suggested that baicalin may protect the cell from pro-inflammatory factors, such as cytokine and chemokine, through disrupting several inflammation-associated signaling pathways inside the cell. This result suggests a potential of baicalin to act as an anti-inflammatory compound that may alleviate cytokine-induced symptoms.

Our study has a number of limitations. All in silico results require further experimental verification. Although the in silico data of baicalin suggested a better fitting score on 3CLpro than PLpro, the relative difference between $\mathrm{IC}_{50}$ values however showed opposite difference. Admittedly, molecular docking may not reflex the actual ligand-protein interactions. However, docking patterns of baicalin on proteases are required for further structural based drug design, for example, baicalin derivatives to improve the specificity and efficacy on targets. Additionally, the effect on targets found in pathway analysis results, such as MAPK, SYK, PI3K, and GSK3, are needed to be further investigated. Furthermore, the metabolic profile of baicalin should be also taken into account in subsequent studies.

\section{Conclusion}

Our study indicated that baicalin, a natural compound that has been used in traditional Chinese medicine with minimal side effects, has a potential therapeutic effect against SARS-CoV-2 infection. Baicalin exhibits a signifi- cant inhibitory effect on PLpro and 3CLpro of SARS$\mathrm{CoV}-2$, possibly by direct binding on the catalytic site of these enzymes. Moreover, baicalin has potential on manipulating inflammation-related pathways that possibly diminishing the "cytokine storm" syndrome which may occur in severe cases of COVID-19. Our findings suggested that continuous research is needed to develop baicalin as a supportive treatment therapeutic for COVID-19.

\section{Acknowledgments}

We thank Kuan-Wen Chen and Tzu-Mao Hung in GGA Corporation, Molecular Science Center (Taiwan) for performing the molecular docking to 3CLpro, PLpro, and RDRP.

\section{Statement of Ethics}

The paper is exempt from Ethics Committee approval since this study uses in silico and in vitro approaches, and no human or animal testing has been conducted.

\section{Conflict of Interest Statement}

The authors declare no conflict of interest.

\section{Funding Sources}

Funding for this study was provided in part by research grants from the China Medical University Hospital, Taichung, Taiwan awarded to Dr. J.-S. Yang (Grant No: DMR-110-136), to Y.-M. Hsu (Grant No: CMU106-S-13) and a grant from the Ministry of Science and Technology, Taiwan awarded, to Dr. J.-S. Yang (MOST 109-2320-B-039-041).

\section{Author Contributions}

C. Lin, H.-A. Ha, F.-J. Tsai, and J.-S. Yang were involved in the design of the study. Y.-M. Hsu, T.-J. Ho, G.-K. Wang, and Y.-J. Chiu performed the experiments. C. Lin, H.-A. Ha, Y.-J. Chiu, and J.-S. Yang drafted the manuscript. C. Lin, F.-J. Tsai, H.-A. Ha, and J.-S. Yang were involved in the revising and editing of the manuscript. All the authors have read and approved the final manuscript.

\section{Data Availability Statement}

All data generated or analyzed during this study are included in this article and its online supplementary material file. Further inquiries can be directed to the corresponding author. 


\section{References}

1 Nicola M, Alsafi Z, Sohrabi C, Kerwan A, AlJabir A, Iosifidis C, et al. The socio-economic implications of the coronavirus pandemic (COVID-19): a review. Int J Surg. 2020 Jun; 78:185-93.

2 Ali I, Alharbi OML. COVID-19: disease, management, treatment, and social impact. Sci Total Environ. 2020 Aug 1;728:138861.

3 Alaran AJ, Adebisi YA, Badmos A, KhalidSalako F, Gaya SK, Ilesanmi EB, et al. Uneven power dynamics must be levelled in COVID-19 vaccines access and distribution. Public Health Pract. 2021;2: 100096.

4 Wibawa T. COVID-19 vaccine research and development: ethical issues. Trop Med Int Health. 2021;26(1):14-9.

5 Madas BG, Füri P, Farkas Á, Nagy A, Czitrovszky A, Balásházy I, et al. Deposition distribution of the new coronavirus (SARS-CoV-2) in the human airways upon exposure to cough-generated droplets and aerosol particles. Sci Rep. 2020;10(1): 22430.

6 Tsai SC, Lu CC, Bau DT, Chiu YJ, Yen YT, Hsu YM, et al. Approaches towards fighting the COVID-19 pandemic (Review). Int J Mol Med. 2021 Jan;47(1):3-22.

7 Jo S, Kim S, Kim DY, Kim MS, Shin DH. Flavonoids with inhibitory activity against SARS-CoV-2 3CLpro. J Enzyme Inhib Med Chem. 2020;35(1):1539-44.

8 Rehman M, AlAjmi MF, Hussain A. Natural compounds as inhibitors of SARSCoV-2 main protease (3CLpro): a molecular docking and simulation approach to combat COVID-19. Curr Pharma Des. 2020 .

9 Wu C, Liu Y, Yang Y, Zhang P, Zhong W, Wang Y, et al. Analysis of therapeutic targets for SARS-CoV-2 and discovery of potential drugs by computational methods. Acta Pharm Sin B. 2020;10(5):766-88.

10 Mishra KP, Singh AK, Singh SB. Hyperinflammation and immune response generation in COVID-19. Neuroimmunomodulation. 2020;27(2):80-6.

11 Conti P, Ronconi G, Caraffa A, Gallenga CE, Ross R, Frydas I, et al. Induction of pro-inflammatory cytokines (IL-1 and IL-6) and lung inflammation by coronavirus-19(COVI19 or SARS-CoV-2): anti-inflammatory strategies. J Biol Regul Homeost Agents. 2020 March;34(2):327-31.

12 Felsenstein S, Herbert JA, McNamara PS, Hedrich CM. COVID-19: immunology and treatment options. Clin Immunol. 2020 Jun; 215:108448.

13 Huang T, Liu Y, Zhang C. Pharmacokinetics and bioavailability enhancement of baicalin: a review. Eur J Drug Metab Pharmacokinet. 2019 Apr;44(2):159-68.

14 Zhang QH, Tan L, Gou Q, Zhou LD, Wang CZ, Yuan CS. 6,8-di-C-glycosyl flavones with $\beta$-furanoarabinose from scutellaria ba- icalensis and their anti-inflammatory activities. Nat Prod Res. 2019 May;33(9):124350 .

15 Ming J, Zhuoneng L, Guangxun Z. Protective role of flavonoid baicalin from scutellaria baicalensis in periodontal disease pathogenesis: a literature review. Complement Ther Med. 2018 Jun;38:11-8.

16 Liao H, Ye J, Gao L, Liu Y. The main bioactive compounds of Scutellaria baicalensis Georgi. for alleviation of inflammatory cytokines: a comprehensive review. Biomed Pharmacother. 2021;133:110917.

17 Leonova GN, Shutikova AL, Lubova VA, Maistrovskaya OS. Inhibitory activity of scutellaria baicalensis flavonoids against tickborne encephalitis virus. Bull Exp Biol Med. 2020 Mar;168(5):665-8.

18 Zandi K, Lim TH, Rahim NA, Shu MH, Teoh BT, Sam SS, et al. Extract of Scutellaria baicalensis inhibits dengue virus replication. BMC Complement Altern Med. 2013 Apr 29; 13:91.

19 Chandra A, Chaudhary M, Qamar I, Singh $\mathrm{N}$, Nain V. In silico identification and validation of natural antiviral compounds as potential inhibitors of SARS-CoV-2 methyltransferase. J Biomol Struct Dyn. 2021 Feb 15:1-11.

20 Liu H, Ye F, Sun Q, Liang H, Li C, Li S, et al. Scutellaria baicalensis extract and baicalein inhibit replication of SARS-CoV-2 and its 3Clike protease in vitro. J Enzyme Inhib Med Chem. 2021;36(1):497-503.

21 Russo M, Moccia S, Spagnuolo C, Tedesco I, Russo GL. Roles of flavonoids against coronavirus infection. Chem Biol Interact. 2020 Sep 1;328:109211

22 Zhong LLD, Lam WC, Yang W, Chan KW, Sze SCW, Miao J, et al. Potential targets for treatment of coronavirus disease 2019 (COVID-19): a review of Qing-Fei-Pai-Du-Tang and its major herbs. Am J Chin Med. 2020; 48(5):1051-71.

23 Fang F, Xie Z, Quan J, Wei X, Wang L, Yang L. Baicalin suppresses Propionibacterium acnes-induced skin inflammation by downregulating the NF- $\kappa \mathrm{B} / \mathrm{MAPK}$ signaling pathway and inhibiting activation of NLRP3 inflammasome. Braz J Med Biol Res. 2020;53: e9949.

24 Zhu L, Shen H, Gu PQ, Liu YJ, Zhang L, Cheng JF. Baicalin alleviates TNBS-induced colitis by inhibiting PI3K/AKT pathway activation. Exp Ther Med. 2020;20(1): 581-90.

25 Fu S, Zhao W, Xiong C, Guo L, Guo J, Qiu $Y$, et al. Baicalin modulates apoptosis via RAGE, MAPK, and AP-1 in vascular endothelial cells during Haemophilus parasuis invasion. Innate Immun. 2019;25(7):42032.

26 Curtis JR, Owensby JK, Xie F. Comparative safety of flavocoxid versus prescription NSAIDs among osteoarthritis patients. Os- teoarthritis Cartilage. 2020 Jul;28(7):91723.

27 Boozari M, Hosseinzadeh H. Natural products for COVID-19 prevention and treatment regarding to previous coronavirus infections and novel studies. Phytother Res. 2021 Feb; 35(2):864-76.

28 Chen KH, Wang SF, Wang SY, Yang YP, Wang ML, Chiou SH, et al. Pharmacological development of the potential adjuvant therapeutic agents against coronavirus disease 2019. J Chin Med Assoc. 2019;83(9): 817-21.

29 Berman HM, Bhat TN, Bourne PE, Feng Z, Gilliland G, Weissig $\mathrm{H}$, et al. The protein data bank and the challenge of structural genomics. Nat Struct Biol. 2000;7(1):957-9.

30 Rut W, Lv Z, Zmudzinski M, Patchett S, Nayak D, Snipas SJ, et al. Activity profiling and crystal structures of inhibitor-bound SARSCoV-2 papain-like protease: a framework for anti-COVID-19 drug design. Sci Adv. 2020 Oct;6(42):4596.

31 Yin W, Mao C, Luan X, Shen DD, Shen Q, Su $\mathrm{H}$, et al. Structural basis for inhibition of the RNA-dependent RNA polymerase from SARS-CoV-2 by remdesivir. Science. 2020 Jun 26;368(6498):1499-504.

32 Jin Z, Du X, Xu Y, Deng Y, Liu M, Zhao Y, et al. Structure of Mpro from SARS-CoV-2 and discovery of its inhibitors. Nature. 2020; 582(7811):289-93

33 Kanehisa M, Furumichi M, Tanabe M, Sato Y, Morishima K. KEGG: new perspectives on genomes, pathways, diseases and drugs. Nucleic Acids Res. 2017 Jan 4;45(D1):D353d61.

34 Sanders JM, Monogue ML, Jodlowski TZ, Cutrell JB. Pharmacologic treatments for coronavirus disease 2019 (COVID-19): a review. JAMA. 2020 May 12;323(18):182436.

35 Wu C, Liu Y, Yang Y, Zhang P, Zhong W, Wang Y, et al. Analysis of therapeutic targets for SARS-CoV-2 and discovery of potential drugs by computational methods. Acta Pharm Sin B. 2020 May;10(5):766-88.

36 Zhang L, Liu J, Cao R, Xu M, Wu Y, Shang W, et al. Comparative antiviral efficacy of viral protease inhibitors against the novel SARSCoV-2 in vitro. Virol Sin. 2020;35(6):776-84.

37 Rajpoot S, Alagumuthu M, Baig MS. Dual targeting of 3CLpro and PLpro of SARS-CoV-2: a novel structure-based design approach to treat COVID-19. Curr Res Struct Biol. 2021; 3:9-18.

38 Krammer A, Kirchhoff PD, Jiang X, Venkatachalam CM, Waldman M. LigScore: a novel scoring function for predicting binding affinities. J Mol Graph Model. 2005 Apr; 23(5):395-407.

39 Zandi K, Musall K, Oo A, Cao D, Liang B, Hassandarvish $\mathrm{P}$, et al. Baicalein and baicalin inhibit SARS-CoV-2 RNA-dependent-RNA polymerase. Microorganisms. 2021;9(5):893. 
40 Wu Y, Wang F, Fan L, Zhang W, Wang T, Du $\mathrm{Y}$, et al. Baicalin alleviates atherosclerosis by relieving oxidative stress and inflammatory responses via inactivating the $\mathrm{NF}-\mathrm{\kappa B}$ and $\mathrm{p} 38$ MAPK signaling pathways. Biomed Pharmacother. 2018 Jan;97:1673-9.

41 Chernyak BV, Popova EN, Prikhodko AS, Grebenchikov OA, Zinovkina LA, Zinovkin RA. COVID-19 and oxidative stress. Biochemistry. 2020;85(12):1543-53.
42 Udrea A-M, Mernea M, Buiu C, Avram S. Scutellaria baicalensis flavones as potent drugs against acute respiratory injury during SARS-CoV-2 infection: structural biology approaches. Processes. 2020;8(11):1468.

$43 \mathrm{Pu}$ WL, Bai RY, Zhou K, Peng YF, Zhang MY, Hottiger MO, et al. Baicalein attenuates pancreatic inflammatory injury through regulating MAPK, STAT 3 and NF- $\kappa B$ activation. Int Immunopharmacol. 2019;72:204-10.
44 Zhang L, Wang X, Wang R, Zheng X, Li N, Li $\mathrm{H}$, et al. Baicalin potentiates TRAIL-induced apoptosis through p38 MAPK activation and intracellular reactive oxygen species production. Mol Med Rep. 2017;16(6):8549-55.

45 Grimes JM, Grimes KV. p38 MAPK inhibition: a promising therapeutic approach for COVID-19. J Mol Cell Cardiol. 2020;144:635. 